\title{
MODELING AND CALCULATION OF THE GLOBAL SOLAR IRRADIANCE ON SLOPES
}

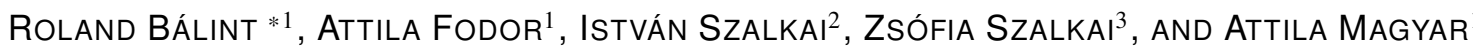 \\ ${ }^{1}$ Department of Electrical Engineering and Information Systems, Faculty of Information Technology, \\ University of Pannonia, Egyetem u. 10, Veszprém, 8200, HUNGARY \\ ${ }^{2}$ Department of Mathematics, Faculty of Information Technology, University of Pannonia, Egyetem u. 10, \\ Veszprém, 8200, HUNGARY \\ ${ }^{3}$ Eötvös Loránd University, Egyetem tér 1-3, Budapest, 1053, HUNGARY
}

\begin{abstract}
The first step with regard to a simple model of a Photovoltaic Power Plant is developed in this paper based on astronomical and engineering principles. A solar irradiance model is presented in this paper that can be used to forecast the solar energy a surface on Earth is exposed to. The obtained model is verified against engineering expectations. The developed model can serve as a basis for forecasting the power of solar energy.
\end{abstract}

Keywords: solar irradiance, modeling, solar geometry, inclined surface

\section{Introduction}

As sustainable development is becoming highly important all over the world, the power production forecast of Photovoltaic Power Plants (PVPPs) is becoming ever more necessary. The Sun is the greatest potential energy source available. The thermal energy it emits can be transformed into electrical energy, or its irradiance can be converted into electricity by solar panels. The amount of energy produced by both of the solutions above is directly dependent on the solar irradiance.

Several works have focused on the determination or forecast of this energy and its dependence on the geometrical parameters of the surface. A general model of the angle of incidence of solar irradiance with regard to the azimuth is derived in Ref. [1] that is used for both fixed and tracking surfaces. In Ref. [2], a simple spectral model is given that is applicable to inclined surfaces and clear skies.

A very good comparative study was proposed in Refs. [3] and [4] where different area-specific models were analysed with regard to the optimal tilt angle. Unfortunately, the Hungarian region is not covered by the models used in this study. A semi-empirical method for short-term (hourly) solar irradiance forecasting was proposed in Ref. [5] founded on the widely used ÅngströmPrescott model.

The aim of this work is to propose a model that is not only able to handle clear-sky conditions but clouds as

*Correspondence: balazs.istvan@virt.uni-pannon.hu well. The computational complexity of the model is also a key factor which should be kept to a minimum.

The organization of this paper is as follows: Section 2 introduces the problem at hand and also presents the motivation of the paper. Afterwards, the elementary astronomical relationships are collected and the proposed model is developed in Section 3. The mathematical model is verified against basic engineering expectations in Section 4. Finally, some concluding remarks are presented.

\section{Problem statement}

The produced energy forecast of the renewable energy sources is one of the main problems of the Distributed Energy Resource Management System. The operators of the electrical grids need precise mathematical models of renewable power plants, e.g. PVPPs, wind farms or hydroelectric power plants. The first step to develop a simple model of a PVPP is presented in this paper based on first astronomical then engineering principles.

The energy production of a PVPP is a function of the following:

- parameters of the PVPP,

- weather (cloud, temperature, wind),

- quality of the atmosphere (dust, relative humidity),

- activity of the Sun (11-year solar cycle).

The parameters of the PVPPs are the following:

- position, 
- orientation of the PV solar panels,

- tilt angle,

- azimuth angle,

- surface area (or the number of PV solar panels),

- nominal power of the PV solar panels,

- efficiency of the solar inverter.

By using the first four parameters, the solar energy can be calculated by the developed solar irradiance model which is presented in this paper.

\section{Astronomical relationships - Sun-Earth geometries}

To estimate the solar irradiance from the Sun, some astronomical (geometrical) calculations to define the position of the Sun relative to the local horizontal surface need to be conducted. Many different equations are found in the literature to calculate the same parameter. The size of these equations, in addition to the frequency of measurements, vary significantly. In this section some equations are compared.

\subsection{Comparison of the available solar geome- try models}

Due to the elliptic orbit of the Earth around the Sun, some parameters exist with a 1-year cycle. These parameters can be calculated with different equations and the difference between values of these equations varies due to discrepancies in the description of the method. The calculations regard a year as consisting of 365 days. Most of the results were obtained from Ref. [6] and references therein.

\section{Relative Sun-Earth distance}

The first parameter is the reciprocal of the square of the Sun-Earth distance during the year. The elliptic orbit of the Earth causes this to change in value. This relative distance can be calculated from equations

$$
\begin{aligned}
E_{0}= & \left(\frac{r_{0}}{r}\right)^{2}=1.000110+ \\
& +0.034221 \cos \left(2 \pi \frac{d_{n}-1}{365}\right)+ \\
& +0.001280 \sin \left(2 \pi \frac{d_{n}-1}{365}\right)+ \\
& +0.000719 \cos \left(4 \pi \frac{d_{n}-1}{365}\right)+ \\
& +0.000077 \sin \left(4 \pi \frac{d_{n}-1}{365}\right)
\end{aligned}
$$

and

$$
E_{0}=\left(\frac{r_{0}}{r}\right)^{2}=1+0.033 \cos \left(2 \pi \frac{d_{n}}{365}\right)
$$

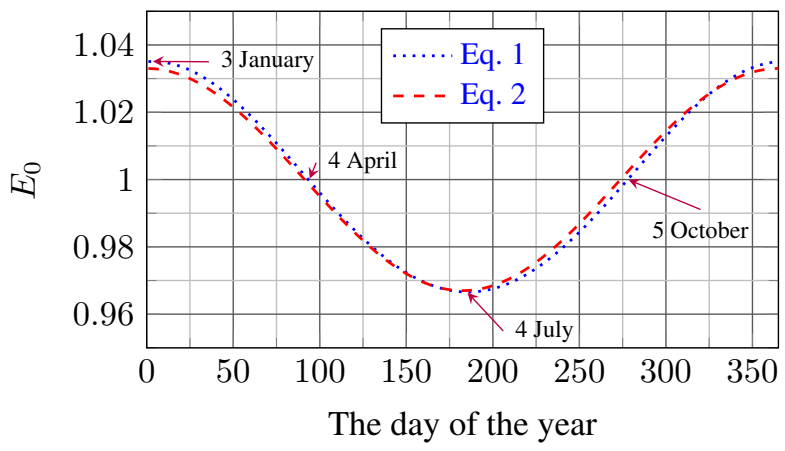

Figure 1: The calculated value of the reciprocal of the square of the Sun-Earth distance $\left(E_{0}\right)$ using Eqs. 1 and 2

Eqs. 1 and 2 are from Refs. [6] and [7]. $E_{0}$ denotes the reciprocal of the square of the Sun-Earth distance, $r_{0}$ represents the mean Sun-Earth distance (1 AU) and $r$ stands for the Sun-Earth distance on the $d_{n}$ th day of the year. Values of $E_{0}$ using various equations are shown in Fig. 1.

This change will change the solar constant too. The solar constant, $I_{0}$, yields the following solar irradiance value (in $\mathrm{W} / \mathrm{m}^{2}$ ) at the edge of the Earth's atmosphere. This constant value is $1367 \mathrm{~W} / \mathrm{m}^{2}$ if the Earth is a distance of $1 \mathrm{AU}$ (astronomical unit) from the Sun. This value is greater at perihelion (minimum Sun-Earth distance $\approx 0.983 \mathrm{AU}$ on 3 January) and less at aphelion (maximum Sun-Earth distance $\approx 1.017 \mathrm{AU}$ on 4 July). Since the irradiance is proportional to the surface area, which is inversely proportional to the square of the distance, the corrected solar irradiance can be calculated from

$$
I_{n}=I_{0} E_{0}
$$

\section{Solar declination}

The declination of the Sun, $\delta$, yields the latitude above which the path of the Sun follows. Therefore, the zenith angle is zero at local noon. This value can be calculated from the following equations [6]:

$$
\begin{aligned}
\delta= & (0.006918- \\
& -0.399912 \cos \left(2 \pi \frac{d_{n}-1}{365}\right)+ \\
& +0.070257 \sin \left(2 \pi \frac{d_{n}-1}{365}\right)- \\
& -0.006758 \cos \left(4 \pi \frac{d_{n}-1}{365}\right)+ \\
& +0.000907 \sin \left(4 \pi \frac{d_{n}-1}{365}\right)- \\
& -0.002697 \cos \left(6 \pi \frac{d_{n}-1}{365}\right)+ \\
& \left.+0.00148 \sin \left(6 \pi \frac{d_{n}-1}{365}\right)\right) \frac{180^{\circ}}{\pi}
\end{aligned}
$$




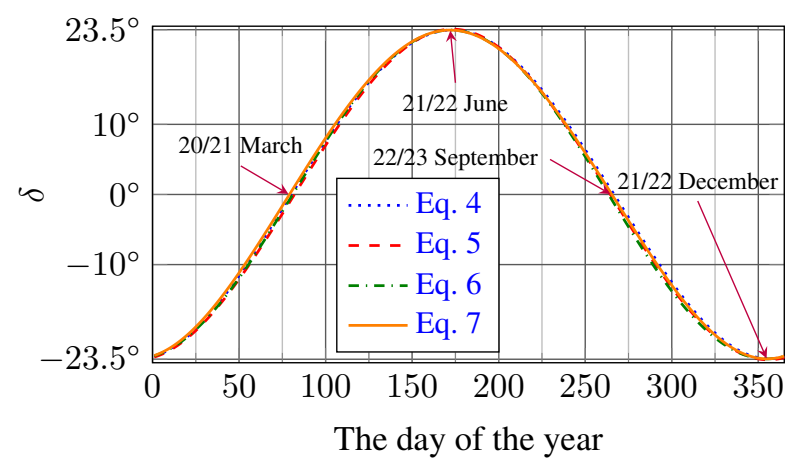

Figure 2: The calculated value of the declination of the Sun $(\delta)$ using Eqs. 4-7

$$
\begin{gathered}
\delta=\arcsin \left(0.4 \sin \left(\frac{360}{365}\left(d_{n}-82\right)\right)\right) \\
\delta=23.45^{\circ} \sin \left(\frac{2 \pi}{365}\left(d_{n}+284\right)\right)
\end{gathered}
$$

and

$$
\begin{aligned}
\delta= & \arcsin \left(0 . 3 9 7 7 9 \operatorname { s i n } \left(4.8888+0.017214 d_{n}+\right.\right. \\
& \left.\left.+0.033 \sin \left(0.017214 d_{n}\right)\right)\right)
\end{aligned}
$$

The results of different equations that calculate the declination of the Sun are shown in Fig. 2. The Sun is above the equator on 20/21 March (vernal equinox) and 22/23 September (autumnal equinox), moreover, the winter solstice is on 21/22 December and the summer solstice on 21/22 June. The difference between the equations in Fig. 2 is minimal. The maximum change in the declination when calculated on a daily basis is less than $0.5^{\circ}\left(\left|\delta\left(d_{n+1}\right)-\delta\left(d_{n}\right)\right|\right)$.

\section{Equation of time}

A solar day varies in length throughout the year. A day on Earth is 24 hours long and is based on the rotation of the Earth around its polar axis. A solar day is based on the rotation of the Earth around its polar axis and on its motion around the Sun. The elliptic orbit of the Earth causes a difference between the time of day and the solar time of day (astronomical). This means that noon in local and astronomical times (the Sun is in the southern hemisphere) differ.

This time difference can be calculated from the following equations [6]:

$$
\begin{aligned}
E_{t}= & {\left[0.000075+0.001868 \cos \left(2 \pi \frac{d_{n}-1}{365}\right)-\right.} \\
& -0.032077 \sin \left(2 \pi \frac{d_{n}-1}{365}\right)- \\
& -0.014615 \cos \left(4 \pi \frac{d_{n}-1}{365}\right)- \\
& \left.-0.04089 \sin \left(4 \pi \frac{d_{n}-1}{365}\right)\right] 229.18
\end{aligned}
$$

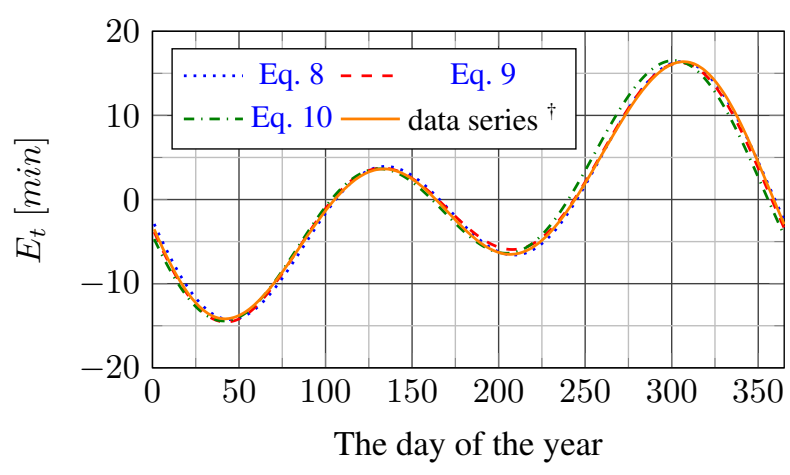

Figure 3: The values of the Equation of Time over a year using Eqs. 8-10 and a series for the year 2018

$$
\begin{aligned}
E_{t}= & {\left[0 . 0 4 3 \operatorname { s i n } \left(2 \left(4.8888+0.017214 d_{n}+\right.\right.\right.} \\
& \left.\left.+0.033 \sin \left(0.017214 d_{n}\right)\right)\right)- \\
& -0.03342 \sin \left(0.017214 d_{n}\right)+ \\
& \left.+0.2618 t_{\mathrm{UTC}}-\pi\right] 229.18
\end{aligned}
$$

and

$$
\begin{aligned}
E_{t}= & -7.655 \sin \left(2 \pi \frac{d_{n}}{365}\right)+ \\
& +9.873 \sin \left(4 \pi \frac{d_{n}}{365}+3.588\right) .
\end{aligned}
$$

Eqs. 8 and 9 yield $E_{t}$ in radians and the constant 229.18 converts this into minutes. This constant can be calculated from

$$
229.18=\frac{360^{\circ}}{2 \pi} \frac{360^{\circ}}{24 \cdot 60 \min } 16 \mathrm{~min}
$$

Fig. 3 shows the results according to Eqs. 8-10 over a whole year, together with astronomical data for the year 2018.

The largest difference was calculated by Eq. 10 when compared with the other data.

\subsection{Solar beam equations}

Using the equations in Section 3.1, the position and angles of the Sun at a local (geological) position can be calculated with the GPS coordinates $\phi_{\text {lat }}$ and $\phi_{\text {long }}$.

Fig. 4 shows the path of the Sun and the angles of the actual position of the Sun. The nomenclature is as follows:

- $\alpha$ : solar altitude angle

- $\theta_{\mathrm{z}}$ : solar zenith angle

- $\psi$ : solar azimuth angle

Since the altitude $(\alpha)$ and zenith angles $\left(\theta_{\mathrm{z}}\right)$ are complementary angles,

$$
\alpha+\theta_{\mathrm{z}}=90^{\circ}
$$

the local solar hour angle can be calculated from [6]

\footnotetext{
†http://www.ppowers.com/EoT.htm
} 


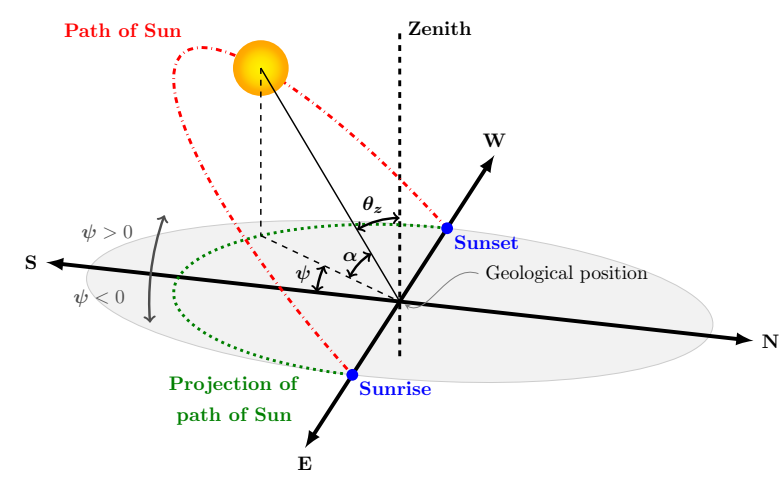

Figure 4: The local position of the Sun with important angles

$$
h=180^{\circ}-15^{\circ}\left(t_{\mathrm{UTC}}+\frac{E_{t}}{60}\right)-\phi_{\text {long }},
$$

where $t_{\mathrm{UTC}}$ is the local time in time zone +0 in hours and $\phi_{\text {long }}$ is the local longitudinal position in degrees.

The cosine of the solar zenith angle can be calculated by using the solar declination, local latitudinal position and solar hour angle. According to Eq. 12,

$$
\begin{aligned}
\cos \left(\theta_{\mathrm{z}}\right) & =\sin (\delta) \sin \left(\phi_{\text {lat }}\right)+ \\
& +\cos (\delta) \cos \left(\phi_{\text {lat }}\right) \cos (h),
\end{aligned}
$$

and

$$
\cos \left(\theta_{\mathrm{z}}\right)=\sin (\alpha),
$$

(Eq. 14 is from Ref. [6]) the solar azimuth angle $(\psi)$ can be calculated from

$$
\cos (\psi)=\frac{\sin (\alpha) \sin \left(\phi_{\text {lat }}\right)-\sin (\delta)}{\cos (\alpha) \cos \left(\phi_{\text {lat }}\right)}
$$

and

$$
\sin (\psi)=\frac{\cos (\delta) \sin (h)}{\cos (\alpha)}
$$

\subsection{Global solar irradiance}

The solar irradiance can be calculated in a simple form:

$$
I=I_{n} q^{T_{\mathrm{m}} z} \sin (\alpha)
$$

where $q$ denotes the clear sky transmissivity through a thickness of one atmosphere, $T_{\mathrm{m}}$ represents the Linke turbidity factor of the air and $z$ stands for the relative path length of the solar beam through the atmosphere. Parameter $q$ is a constant with a value of 0.93 (when the sky is dry and clear and the zenith angle is equal to zero). The value of $T_{\mathrm{m}}$ determines how many clear atmospheres should be stacked to achieve the actual spectral transmittance. This value depends on the amount of water vapor, dust, smog, etc. In the EU, $T_{\mathrm{m}}$ is between 1.8 and 4 . The suggested formula for the calculation of parameter $z$ is

$$
z=\frac{l_{\text {atm }}}{R_{\text {Earth }}-r_{\text {Earth }}},
$$

where $l_{\text {atm }}$ denotes the path length of the solar beam through the atmosphere, $r_{\text {Earth }}$ represents the mean radius of the Earth's surface and $R_{\text {Earth }}$ stands for the radius of the Earth when the atmosphere is included $\left(R_{\text {Earth }}-r_{\text {Earth }}\right.$ is the thickness of the atmosphere). The value of $l_{\text {atm }}$ depends on the solar altitude and can be calculated using the law of cosines which yields

$$
\begin{gathered}
l_{\mathrm{atm}}^{2}-2 r_{\text {Earth }} l_{\mathrm{atm}} \cos \left(\alpha+90^{\circ}\right)+ \\
+\left(r_{\text {Earth }}^{2}-R_{\text {Earth }}^{2}\right)=0 .
\end{gathered}
$$

The correct value of $l_{\mathrm{atm}}$ is the positive root of Eq. 20 .

Eq. 18 yields the solar irradiance under ideal conditions. However, aerosols in the atmosphere cause diffuse scattering and absorb a proportion of the solar irradiance. Furthermore, clouds also have an effect on the irradiance. Some models divide the global solar irradiance into two parts:

- Direct solar irradiance

- Diffuse solar irradiance

\section{Irradiance on a horizontal surface}

Various models exist to calculate the global solar irradiance on a horizontal surface, e.g., a Hungarian model [8] is presented in

$$
G=\left(I_{n} \sin (\alpha)+a\right)\left(1+b_{1} N^{b_{2}}\right),
$$

where $I_{n}$ denotes the corrected solar constant, $N$ represents the cloudy parameter between 0 and 1 ( 0 when clear, 1 when overcast). $a$ is a local correction value (a negative constant in $\mathrm{W} / \mathrm{m}^{2}$, and $b_{1}$ as well as $b_{2}$ are locally constant in terms of the cloud correction.

This Hungarian model [8] takes the cloud cover into consideration but not the transparency of the atmosphere $\left(q, T_{\mathrm{m}}\right.$ and $\left.l_{\mathrm{atm}}\right)$. A German model [9] accounts for atmospheric conditions. Equation

$$
G=I_{n} \sin (\alpha) A_{d} e^{-B_{d} T_{\mathrm{m}} z}\left(1-a_{d} N^{b_{d}}\right)
$$

shows the global solar irradiance and equation

$$
S=I_{n} \sin (\alpha) A_{d} q^{T_{\mathrm{m}} z}\left(1-N^{b_{d}}\right)
$$

presents the direct solar irradiance formula. The parameters $a_{d}, b_{d}, A_{d}$ and $B_{d}$ are place-dependent values. A typical parameter set is $a_{d}=0.72, b_{d}=3.2, A_{d}=0.7$ and $B_{d}=0.027$. The diffuse solar irradiance can be calculated by a simple subtraction.

$$
D=G-S
$$

The calculated global solar irradiance is shown in Fig. 5 over a year with weekly resolutions during clear sky conditions in Veszprém, Hungary. The red curve tracks the maximum values of the astronomical noons on each curve (analemma curve). This curve shows the effect of 


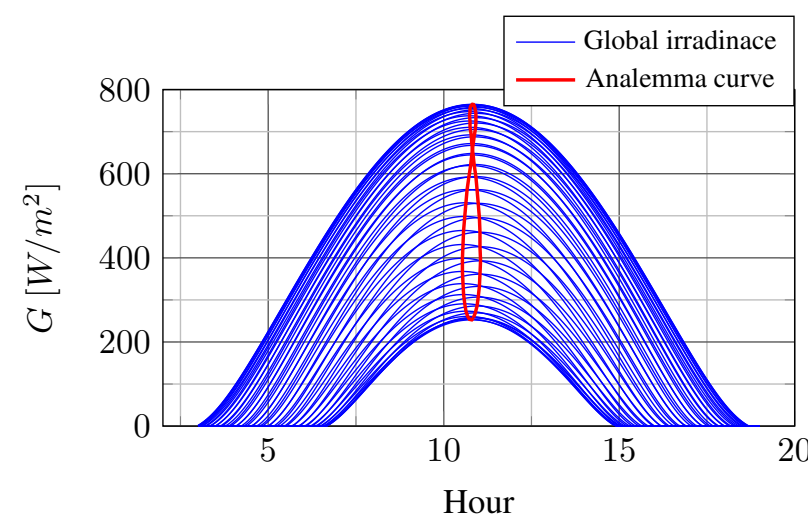

Figure 5: Calculated global solar irradiance on a horizontal surface over the year in Veszprém according to weekly resolutions and the analemma curve (astronomical noon times)

the $E_{t}$, the duration of which is approximately 11 hours because the longitudinal position of Veszprém is $17.9^{\circ}$ and this causes a delay of about 1 hour (the local time zone is +1 ).

Fig. 5 clearly illustrates the difference in irradiance between the winter and summer periods.

\section{Irradiance on the slope}

For the sake of simplicity the Cartesian coordinate system is used, the axes of which are S (South), W (West) and $\mathrm{Z}$ (Zenith). The azimuth angle $\psi$ in the direction $\mathrm{S}$ W-N-E-S and the altitude of the Sun $\alpha$ have been calculated, so the normed vector that points towards the Sun is $\overrightarrow{O S}=[\cos (\alpha) \cos (\psi), \cos (\alpha) \sin (\psi), \sin (\alpha)]^{\top}$. If the surface $\Sigma$ exhibits an angle of inclination of $\beta$ to the horizon and an orientation of $\gamma$, which defines $\gamma$ as negative, positive or zero if and only if the surface faces SE, SW or S, respectively, then the normed vector of the surface (contained within the same half-space as the Sun) is $\vec{n}$ and exhibits the altitude $\nu=90^{\circ}-\beta$ so $\vec{n}=[\cos (\nu) \cos (\gamma), \cos (\nu) \sin (\gamma), \sin (\nu)]^{\top}$. The angle between $\overrightarrow{O S}$ and $\vec{n}$ is

$$
\begin{aligned}
\theta_{\text {surf }}= & \arccos (\cos (\alpha) \cos (\psi) \cos (\nu) \cos (\gamma)+ \\
& +\cos (\alpha) \sin (\psi) \cos (\nu) \sin (\gamma)+ \\
& +\sin (\alpha) \sin (\nu)) \\
= & \arccos (\cos (\alpha) \cos (\nu)(\cos (\psi) \cos (\gamma)+ \\
& +\sin (\psi) \sin (\gamma))+\sin (\alpha) \sin (\nu))
\end{aligned}
$$

since $\overrightarrow{O S}$ and $\vec{n}$ are normed. So $\overrightarrow{O S}$ meets $\Sigma$ at the angle $\alpha_{\text {surf }}=90^{\circ}-\theta_{\text {surf }}$.

Eq. 23 yields the direct solar irradiance on a horizontal surface with solar altitude angle $\alpha$. The solar altitude

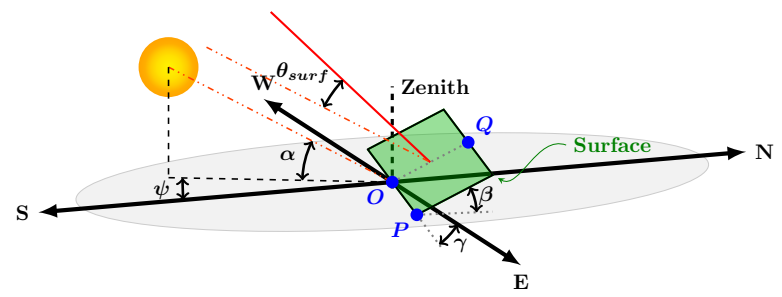

Figure 6: The angle of the solar beam and the zenith angle between the surface and the slope $\beta$ and orientation $\gamma$

angle on the slope is $\alpha_{\text {surf }}$. To calculate the corrected direct solar irradiance, equation

$$
S_{\text {surf }}=S \frac{\sin \left(\alpha_{\text {surf }}\right)}{\sin (\alpha)}
$$

must be used. Calculation of the diffuse solar irradiance is far from trivial. If the diffuse solar irradiance is consistent with the all-sky, equation

$$
D_{\text {surf }}=D \frac{1+\cos (\beta)}{2}
$$

can be used to estimate the value of the irradiance. The global solar irradiance of the slope is the sum of the direct and diffuse solar irradiances:

$$
G_{\text {surf }}=S_{\text {surf }}+D_{\text {surf }} .
$$

\section{Model verification}

The aim of this section is to verify the solar irradiance model proposed in the previous section against basic engineering expectations. Figs. 7 and 8 show the results of the implemented model on 1 March $\left(d_{n}=60\right)$ and 1 July $\left(d_{n}=121\right)$ and the geographical position of Veszprém, Hungary. Both figures present the value of the solar irradiance for various orientations as well as the tilt and azimuth angles.

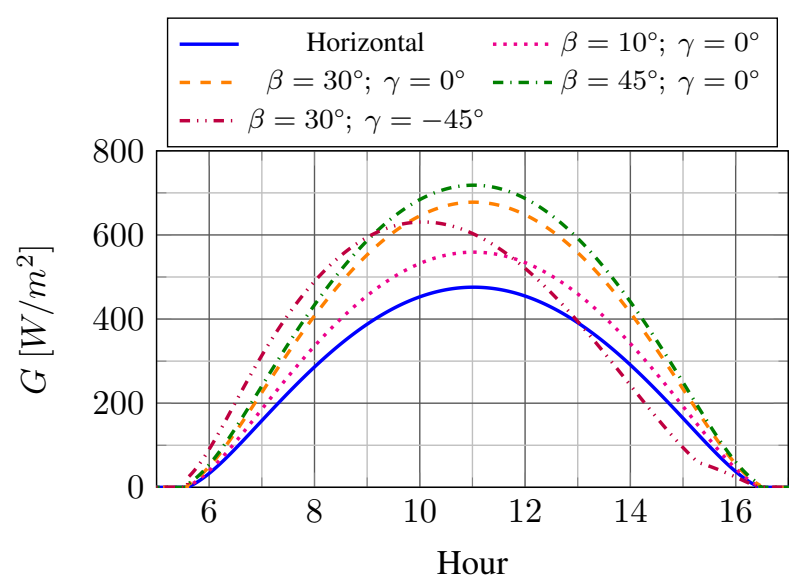

Figure 7: Calculated global solar irradiance on sloping terrain of various orientations on 1 March in Veszprém, Hungary 


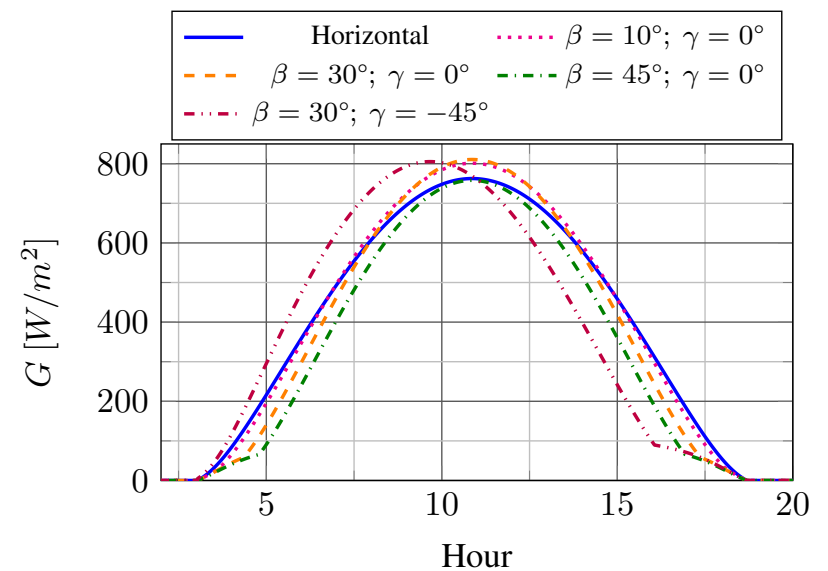

Figure 8: Calculated global solar irradiance on sloping terrain of various orientations on 1 July in Veszprém, Hungary

Irradiance on a horizontal surface is denoted by a blue solid line. The other curves show the value of the solar power with a non-zero tilt angle: $10^{\circ}$ (magenta dotted line), $30^{\circ}$ (orange dashed line with an azimuth angle of zero, purple dashed dotted line with an azimuth angle of $-45^{\circ}$ (South-East)) and $45^{\circ}$ (green dashed dotted line).

The peak values of the curves in Fig. 7 correlate with the tilt angle $(\beta)$. As the tilt angle increases, so does the irradiance when the azimuth angle is constant $\left(\gamma=0^{\circ}\right)$. As the azimuth angle changes towards the southeast, the peak of the curve shifts towards the sunrise.

The effect of the tilt angle is somewhat different in Fig. 8. On 1 July, the absolute peak can be observed for cases $\beta=10^{\circ}$ and $\beta=30^{\circ}$ but the irradiance for the whole day is higher if the tilt angle is smaller. This effect is caused by the angle of the solar altitude (smaller zenith angle). If the tilt angle is greater than the minimum horizontal zenith angle, the solar irradiance will decrease on the slope. On 1 March the maximum angle of the solar altitude did not reach $45^{\circ}$. In other words, a greater tilt angle generates more power on sloping terrain. The effect of the azimuth angle can be seen in Fig. 7.

\section{Conclusions}

A solar irradiance model is presented in this work that can be used for forecasting the solar energy absorbed by an inclined surface, e.g. a PVPP. The developed model is preliminary in nature and serves as a basis for further developments, e.g. forecasting the power of the solar energy under cloudy conditions. The novel element of the model is that the relative path length of the solar beam in the atmosphere can be calculated more accurately. The calculated irradiance an inclined surface is exposed to is another novel element. The proposed model has been verified against engineering expectations and the results show that it works well.

The next step will be the validation of the model using real measured parameters and meteorological data.
By extending the model with an electrical power module, it will also be possible to forecast the amount of electrical energy produced.

\section{Acknowledgement}

We acknowledge the financial support of Széchenyi 2020 under the EFOP-3.6.1-16-2016-00015. We acknowledge the financial support of Széchenyi 2020 under the GINOP-2.2.1-15-2017-00038. A. Magyar was supported by the János Bolyai Research Scholarship of the Hungarian Academy of Sciences.

\section{REFERENCES}

[1] Braun, J.E.; Mitchell, J.C.: Solar geometry for fixed and tracking surfaces, Sol. Energy, 1983 31(5), 439444, DOI: 10.1016/0038-092X(83)90046-4

[2] Bird, R.E.; Riordan, C.: Simple solar spectral model for direct and diffuse irradiance on horizontal and tilted planes at the earth's surface for cloudless atmospheres, J. Clim. Appl. Meteorol., 1986 25(1), 87-97, DOI: 10.1175/15200450(1986)025\%3C0087:SSSMFD\%3E2.0.CO;2

[3] Danandeh, M.A.; Mousavi G., S.M.: Solar irradiance estimation models and optimum tilt angle approaches: A comparative study, Renew. Sust. Energy Rev., 2018 92, 319-330, DOI: 10.1016/j.rser.2018.05.004

[4] Li, D.H.W.; Chau, T.C.; Wan, K.K.W.: A review of the CIE general sky classification approaches, Renew. Sust. Energy Rev., 2014 31, 563-574, DOI: 10.1016/j.rser.2013.12.018

[5] Akarslan, E.; Hocaoglu, F.O.; Edizkan, R.: Novel short term solar irradiance forecasting models, Renew. Energy, 2018 123, 58-66, DOI: 10.1016/j.renene.2018.02.048

[6] Iqbal, M.: An introduction to solar radiation (Academic Press, London, UK), 1st edn., 1983, DOI: 10.1016/B978-0-12-373750-2.X5001-0, ISBN: 978-0-12373750-2

[7] Duffie, J.A.; Beckman, W.A.: Solar engineering of thermal processes (John Wiley \& Sons, New Jersey, USA), 2013, DOI: 10.1002/9781118671603 ISBN:978-0-47087366-3

[8] Práger, T.; Ács, F.; Baranka, G.; Feketéné Nárai, K.; Mészáros, R.; Szepesi, D.; Weidinger, T.: A légszennyezõ anyagok transzmissziós szabványainak korszerũsítése III. fázis Részjelentés 2., 1999

[9] Kasten, F.: Strahlungsaustausch zwischen Oberflächen und Atmosphäre, VDI-Bericht, 1989 (Nr. 721. S.), $131-158$ 


\section{Appendix: Nomenclature}

\begin{tabular}{|c|c|c|}
\hline Symbol & Description & Value/dimension \\
\hline$E_{0}$ & Reciprocal of the relative Sun-Earth squared distance & $\left(r_{0} / r\right)^{2}$ \\
\hline$r_{0}$ & Mean Sun-Earth distance (Astronomical Unit: $[\mathrm{AU}]$ ) & $1 \mathrm{AU}$ \\
\hline$r$ & Actual Sun-Earth distance in $\mathrm{AU}$ & {$[\mathrm{AU}]$} \\
\hline$d_{n}$ & The day of the year & $1-365$ \\
\hline$I_{0}$ & Solar constant & $1367 \mathrm{~W} / \mathrm{m}^{2}$ \\
\hline$I_{n}$ & Corrected solar constant & $I_{0} E_{0}$ \\
\hline$\delta$ & Sun's declination & $\pm 23.5^{\circ}$ \\
\hline$E_{t}$ & Equation of time & $\sim \pm 15 \min$ \\
\hline$t_{\mathrm{UTC}}$ & The time in UTC & $0-24$ \\
\hline$\phi_{\text {lat }}$ & Geographic latitude & $\pm 90^{\circ}$ \\
\hline$\phi_{\text {long }}$ & Geographic longitude & $\pm 180^{\circ}$ \\
\hline$\alpha$ & The angle of the solar altitude & $0^{\circ}-90^{\circ}$ \\
\hline$\theta_{\mathrm{z}}$ & Solar zenith angle & $90^{\circ}-\alpha$ \\
\hline$\psi$ & Solar azimuth angle & $\pm 180^{\circ}$ \\
\hline$h$ & The solar hour angle & $0^{\circ}-360^{\circ}$ \\
\hline$q$ & Clear sky transmissivity & 0.93 \\
\hline$T_{\mathrm{m}}$ & Linke turbidity factor & 1.8-4 in Central EU \\
\hline$z$ & Relative solar beam length in the atmosphere & \\
\hline$l_{\text {atm }}$ & Length of the path of the solar beam through the atmosphere & {$[\mathrm{km}]$} \\
\hline$R_{\text {Earth }}$ & Median radius of the Earth including the atmosphere & $\sim 6470 \mathrm{~km}$ \\
\hline$r_{\text {Earth }}$ & Median radius of the Earth from the ground & $\sim 6370 \mathrm{~km}$ \\
\hline$G$ & Global solar irradiance & {$\left[\mathrm{W} / \mathrm{m}^{2}\right]$} \\
\hline$N$ & Cloudy parameter & $0-1$ \\
\hline$A_{d}$ & Place-dependent constant & \\
\hline$B_{d}$ & Place-dependent constant & \\
\hline$a_{d}$ & Place-dependent constant & \\
\hline$b_{d}$ & Place-dependent constant & \\
\hline$D$ & Diffuse solar irradiance & {$\left[\mathrm{W} / \mathrm{m}^{2}\right]$} \\
\hline$S$ & Direct solar irradiance & {$\left[\mathrm{W} / \mathrm{m}^{2}\right]$} \\
\hline$\beta$ & Angle above the surface from the horizontal position & $0^{\circ}-90^{\circ}$ \\
\hline$\gamma$ & Surface azimuth angle & $\pm 90^{\circ}$ \\
\hline$\theta_{\text {surf }}$ & The solar zenith angle on the surface & $90^{\circ}-\alpha_{\text {surf }}$ \\
\hline$\alpha_{\text {surf }}$ & The angle of the solar altitude on the surface & $0^{\circ}-90^{\circ}$ \\
\hline$S_{\text {surf }}$ & Direct solar irradiance on the surface & {$\left[\mathrm{W} / \mathrm{m}^{2}\right]$} \\
\hline$D_{\text {surf }}$ & Diffuse solar irradiance on the surface & {$\left[\mathrm{W} / \mathrm{m}^{2}\right]$} \\
\hline$G_{\text {surf }}$ & Global solar irradiance on the surface & {$\left[\mathrm{W} / \mathrm{m}^{2}\right]$} \\
\hline
\end{tabular}

